\title{
Depression Symptoms and Risk Factors in Adult Emergency Department Patients: A Multisite Cross-Sectional Prevalence Survey
}

\author{
Nancy Khav, ${ }^{1,2}$ Tracey J. Weiland, ${ }^{1,2}$ George A. Jelinek, ${ }^{1,2}$ \\ Jonathan C. Knott, ${ }^{3}$ and Michael Salzberg, \\ ${ }^{1}$ Emergency Practice and Innovation Centre, St. Vincent's Hospital Melbourne, Victoria Parade, Fitzroy, VIC 3068, Australia \\ ${ }^{2}$ Department of Medicine, The University of Melbourne, Grattan Street, Parkville, VIC 3010, Australia \\ ${ }^{3}$ Emergency Department, The Royal Melbourne Hospital, Grattan Street, Parkville, VIC 3050, Australia \\ ${ }^{4}$ Mental Health Service, St. Vincent's Hospital Melbourne, Victoria Parade, Fitzroy, VIC 3068, Australia \\ Correspondence should be addressed to Tracey J. Weiland; tracey.weiland@svhm.org.au
}

Received 17 June 2013; Accepted 31 July 2013

Academic Editors: L. V. Downey and W. Ryotaro

Copyright (C) 2013 Nancy Khav et al. This is an open access article distributed under the Creative Commons Attribution License, which permits unrestricted use, distribution, and reproduction in any medium, provided the original work is properly cited.

\begin{abstract}
Objectives. To identify the proportion of adult emergency department (ED) patients who screen positive for depression. Secondary aims were to identify factors associated with a positive depression screen and determine predictors of a positive depression screen. Methods. This cross-sectional, prevalence survey of ED patients was conducted at two inner-city hospitals. 350 ED patients were screened for depression using the Patient Health Questionnaire-9 (PHQ-9). Clinical and demographic risk factors were examined through medical records and additional questionnaires. Results. Of 350 participants screened, 50 (14.3\%; 95\% CI = 11.0-18.4\%) screened positive. Independent predictors of depression risk included self-reported depression and/or a previous diagnosis of depression $(\mathrm{OR}=8.345 ; 95 \% \mathrm{CI}=3.524-19.762)$, seeing a mental health service provider in the past 6 months $(\mathrm{OR}=4.518 ; 95 \% \mathrm{CI}=$ 2.107-9.690), and previous discussion about mental health with a local doctor $(\mathrm{OR}=2.369 ; 95 \% \mathrm{CI}=1.025-5.475)$. Conclusion. $\mathrm{ED}$ patients were found to be at a higher risk of depression than the general population. ED-based depression screening, particularly of high-risk populations, has the potential to increase case detection rates and allow earlier management of these patients. Further research and validation of an ED-based depression screening tool are required.
\end{abstract}

\section{Introduction}

It is important to detect depression in the emergency department (ED). Depression may contribute to the clinical presentation via self-harm or somatisation; it may be an important focus for post-ED care, and even though its management is not usually the responsibility of the ED, ED staff can have an important role in informing patients of the nature of their problem and engaging them in appropriate care; and, like all health care sectors, the ED can contribute to the public health task of detecting depression, which is known to be widely underrecognised and undertreated. In detecting depression, the clinical assessment is primary, but screening using validated depression rating scales is an important complementary method.
Depression is highly prevalent in the general population but almost certainly of greater prevalence in the ED population. In the general population, depression is the second leading cause of disability worldwide [1], a significant risk factor for suicide [1], and associated with increased comorbidity and mortality $[2,3]$, decreased adherence to medication and treatment [4], higher healthcare utilization and costs [4], and reduced quality of life [5]. In Australia, depression prevalence in adults is approximately 6\% [6], with many more undetected [6]. Lifetime prevalence is reportedly $20 \%$ [7]. There are good reasons to expect rates to be higher in the ED population [8-12], with higher rates of risk factors and comorbidities [8, 11-13]; advancing age; and higher levels of pain, substance abuse, and rates of self-harm [8, 11-13]. 
In line with such expectations, internationally, one-fifth to one-third of the ED adult population screens positive for depression [8-11, 13]. However, prevalence data for Australian EDs are scarce as are studies of this problem generally. One study documented that $20 \%$ of participants reported feelings of anxiety or depression [14]. Around 3-5\% of annual ED presentations across Australia are mental health related [15, 16 , and $76 \%$ of patients with depressed mood had a history of at least one visit to the ED every six months [17]. Approximately $12 \%$ of ED patients who were depressed reported a past suicide attempt [9]. This is important given the repeated finding that nearly $75 \%$ who die by suicide have seen a medical professional in the last year of their life (including medical professionals in the ED setting) [18]; however, less than a third received mental health treatment [18].

Currently, most Australian EDs lack a specific routine depression screening protocol, with mental health assessments geared toward those presenting with an overt mental health complaint rather than towards those with primarily a physical complaint. Tools validated specifically for EDbased depression screening are unavailable. Without routine screening procedures, depression is poorly detected by ED physicians unless patients present with depression or suicidal ideation as the chief complaint $[12,17]$. Consequently, underlying depression often goes undetected and untreated, as ED staff are accustomed to an acute medical focus, dealing with physical demands first [19].

ED-based depression screening appears warranted. Screening for depression among these high-risk individuals may augment clinical assessment, allowing for proper and early referral to general practitioners and/or specialist mental health services for further definitive management. Early treatment of depression may reduce or avert the development or progression of associated comorbid conditions, thereby reducing the burden of disease on patients and the healthcare system.

Prior to implementing depression screening and referral programmes in the ED, it is important to know the prevalence of the disorder in this setting. Thus the primary aim of this initial study was to estimate the prevalence of Australian ED patients at risk of having depression. Secondary aims were to compare the estimated proportion to that reported for the general population, to identify associated clinical and demographic risk factors for depression, and to determine variables predictive of a positive screen in this population.

\section{Methods}

2.1. Study Design, Setting, and Ethics Approval. A multisite cross-sectional prevalence survey of depression risk in adult ED patients was undertaken in the EDs of two metropolitan adult tertiary referral hospitals in Melbourne, Australia. The population of one hospital is culturally diverse and socially disadvantaged, with high rates of drug and alcohol use among patients, while the other participating site is a major trauma centre. These EDs receive approximately 40,000 and 58,000 annual attendances, respectively. Ethics approval was obtained from the Human Research Ethics Committees of each hospital. A condition of ethics approval was that patients with deliberate self-harm be excluded.

\subsection{Participants}

Inclusion Criteria. Patients presenting to the EDs at either of the two participating sites during the sampling period were eligible to participate.

Exclusion Criteria. Patients aged less than 18, unable to adequately communicate in English, cognitively impaired, or medically unstable were excluded. Patients who were agitated, intoxicated, adversely affected by drugs, or who presented with self-harm were excluded to avoid exacerbation of acute behavioural disturbance or self-harm.

\subsection{Measurement Instruments}

Patient Health Questionnaire (PHQ). The Patient Health Questionnaire-9 (PHQ-9) consists of nine items and is used as a diagnostic and severity measure for depression in primary care [20]. Items cover five categories: depressed mood; fatigue; worthless guilt; concentration and agitation; and suicidal ideation. Patients rate symptoms occurring in the past two weeks on a four-point scale: not at all (0), several days (1), more than half the days (2), and nearly everyday (3). Item scores are summed and categorized into negative (0-4), mild depression (5-9), moderate depression (10-14), moderately severe depression (15-19), and severe major depression (20 or more) [20]. Patients with major depression rarely score less than 10 [20], and thus a score of 10 or more was considered a priori as a positive screen. At this cutoff, the PHQ-9 has a sensitivity of $88 \%$ and specificity of $88 \%$ for major depression in a primary care setting and a positive likelihood ratio of 7.1 $[20,21]$. The PHQ-9 has high diagnostic accuracy for major depression, with an area under curve (AUC) value of 0.95, and for any depressive disorder an AUC of 0.90 [22].

Self-Administered Comorbidities Questionnaire (SCQ). The Self-Administered Comorbidities Questionnaire (SCQ) is a 14-item measure of comorbidity [23] with high test-retest reliability and moderately strong associations with a standard medical record-based comorbidity measure $(r=0.55)$ [23]. It embodies 13 common medical conditions, with an additional item allowing respondents to list unspecified conditions. Participants rated presence of disease (yes/no). Each item was analysed separately.

Personal and Family History Questions. A researcher-devised 14-item question series on personal and family history was administered to determine participants' mental health history and risk factors, which was used in analysis for predictors of depression risk. A binary response format (yes/no) was used for personal history items (previous diagnoses of mental health problems; current use of antidepressants; utilisation of mental health services; and alcohol and tobacco use). For items related to family history of mental health, the response format was multicategory (yes/no/not sure). This tool has not been previously validated. 
2.4. Sample Size. The primary outcome was the proportion of participants screening positive. The prevalence of depression risk in ED patients was expected to be higher than the general population (6\%) [6], due to higher prevalence and levels of risk factors [11-13]. Based on data which indicated a depression risk prevalence of $32 \%$ in ED patients $[8,9]$, the maximum prevalence of depression risk in ED patients was anticipated to be $35 \%$. A sample size of 350 was required to estimate a depression risk prevalence of $35 \%$ to a confidence interval of $95 \%$ and a margin of error of 5\% [24]. This sample size was also calculated as being sufficient to detect a minimum of an $11 \%$ difference between two groups with power set at $80 \%$ and alpha set at 0.05 . Using the standard rule of thumb of 10 cases per predictor, a sample of 350 permitted the inclusion of up to 12 variables in multiple logistic regression analyses.

2.5. Sampling. The proportion of participants recruited from each ED was adjusted to reflect the differing annual attendance rate at each ED for the preceding 12 months. Quota sampling was used whereby patients were recruited according to clinical acuity using the Australasian Triage Scale (ATS). The percentage of patients recruited reflected the distribution in each triage category for each hospital in the preceding 12 months. This sampling strategy ensured a sample representative of the population in terms of acuity.

Sampling was undertaken by a single researcher from $11 / 10 / 2010$ to $31 / 3 / 2011$, between the hours of 9:00 a.m. and 6:00 p.m., and included weekdays and weekends. It was logistically unrealistic to recruit a consecutive sample. Once the quota for each triage category was reached, sampling ceased.

2.6. Procedure. Patients attending either ED during the sampling period were considered for recruitment. For those aged over 18, their eligibility and capacity to consent according to the inclusion and exclusion criteria were determined by the treating clinician. Eligible patients were approached by the researcher, who provided a patient information, consent form, and a verbal explanation of the study. Consenting participants were administered, in order, by the PHQ-9, SCQ, and personal and family history questions.

Participants scoring 10 or more on the PHQ-9 were deemed to have screened positive (primary outcome). The treating doctor was advised of participants that screened positive and determined treatment needs and management (including referral). Where possible, the researcher notified the participant's preferred local doctor of the positive screen via letter.

Further clinical and demographic data were retrieved through electronic medical records allowing collection of age; sex; marital status; country of birth; socioeconomic status (pension/health care card holder); mode of arrival; ATS code; presenting complaint; discharge diagnosis; further admission; and ED length of stay.

2.7. Data Analysis. Data were analysed using Predictive Analytics Software (PASW) Statistics 18 (Chicago, IL). The frequency and percentage (95\% CI) of participants scoring in each classification group were calculated. The prevalence of positive screens in the sample population and the prevalence
TABLE 1: Distribution of patients excluded from the study.

\begin{tabular}{|c|c|c|}
\hline Reason for exclusion & $n$ & $\%$ \\
\hline Agitated & 17 & 2.0 \\
\hline $\begin{array}{l}\text { Altered consciousness or cognitive } \\
\text { impairment }\end{array}$ & 133 & 16.0 \\
\hline Affected by alcohol & 79 & 9.5 \\
\hline Affected by drugs & 37 & 4.5 \\
\hline Cannot communicate in English & 271 & 32.6 \\
\hline Presented with self-harm & 95 & 11.4 \\
\hline Under 18 years old & 7 & 0.8 \\
\hline Unwell & 53 & 6.4 \\
\hline Total ineligible & 692 & 83.3 \\
\hline Refused consent & 135 & 16.2 \\
\hline Withdrew consent ${ }^{*}$ & 4 & 0.5 \\
\hline Total excluded & 831 & 100 \\
\hline
\end{tabular}

* 1 became unwell, 1 discharged before survey completion, and 2 changed their minds.

of depression in the general population as documented in the literature [25] were compared using the $Z$-test for independent proportions. Frequencies and percentages were calculated for clinical and demographic data, with the exception of continuous variables which were summarized using median (IQR) after assessing normality.

Clinical and demographic variables associated with a positive screen on each PHQ scale were explored using Fisher's exact test (for two-by-two contingency tables), Pearson's chi square test, or the Mann-Whitney $U$ test (for continuous data which violated normality).

Variables identified as having significant associations with depression risk on univariate analyses $(P<0.05)$ were tested for intercorrelations prior to inclusion in backward stepwise logistic regression analyses. Variables were only included in the model when the correlation was $r<0.7$. Eleven variables were entered in the final analysis where the probability for inclusion was 0.05 and the probability for exclusion of variables was 0.10 . Odds ratio $(95 \% \mathrm{CI})$ and goodness of fit are reported for the final model.

\section{Results}

3.1. Participation. A total of 1,181 patients $(580 ; 601)$ were considered for eligibility. Of these, $692(58.6 \%)$ were deemed ineligible and excluded (Table 1), leaving 489 patients (209; 280 ) approached. Overall, 350 patients were recruited across the two hospitals $(144 ; 206)$, a participation rate of $71.6 \%$.

3.2. Demographics. The study sample was comparable to data obtained for the preceding year at each site for most demographic variables (Table 2). Demographics are summarized in Table 3.

The majority of participants reported presence of at least one medical condition according to the SCQ. A large proportion of participants also self-reported depressive symptoms ( $n=119 / 350 ; 34.0 \%)$ or had a previous depression diagnosis $(n=114 / 350 ; 32.6 \%)$. 
TABLE 2: Comparison of sample to data for each site for the recruitment period.

\begin{tabular}{lcccc}
\hline & Hospital A & Hospital A & Hospital B & Hospital B \\
& Sample population & Population & Sample population & Population \\
\hline Age, median (IQR) & $50.0(33.0-66.0)$ & $44.0(29.0-65.0)$ & $48.5(32.0-69.0)$ & $43.0(28.0-65.0)$ \\
Gender (male), $n(\%)$ & $76(52.8 \%)$ & $7519(53.8 \%)$ & $108(52.4 \%)$ & $4287(54.7 \%)$ \\
Arrival (ambulance), $n(\%)$ & $34(23.6 \%)$ & $4555(32.6 \%)$ & $116(56.3 \%)$ & $2869(36.6 \%)$ \\
Modal presenting complaint & Abdominal pain & Chest pain & Trauma & Trauma \\
\hline
\end{tabular}

3.3. Depression Screen. Completion of the depression screen took approximately 3 minutes per patient. Of the 350 participants screened for depression using the PHQ-9, $14.3 \%$ ( $n=50 / 350 ; 95 \% \mathrm{CI}=11.0-18.4 \%)$ screened positive, which is significantly greater than the reported prevalence for the general population $(P<0.05)$. The percentages of participants scoring in each classification group are shown in Table 4.

The association of clinical and demographic factors to depression risk for the PHQ-9, as well as its significance, is shown in Table 5.

A large proportion of participants who screened positive for depression also self-reported depression or had been previously diagnosed. Detection rate among these suspected groups, however, was low. Of those who self-reported depression and those who had a previous depression diagnosis, $64.7 \%(n=77 / 119 ; 95 \% \mathrm{CI}=55.8-72.7 \%)$ and $64.0 \%(n=$ $73 / 114 ; 95 \% \mathrm{CI}=54.9-72.3 \%)$, respectively, screened negative on the PHQ-9. In addition, $61.7 \%(n=58 / 94 ; 95 \% \mathrm{CI}=$ $51.6-70.9 \%)$ of participants who reported treatment for depression also screened negative on the PHQ-9, with $63.0 \%$ ( $n=33 / 54 ; 95 \% \mathrm{CI}=47.8-73.0 \%)$ who used antidepressant and $53.3 \%(n=32 / 60 ; 95 \% \mathrm{CI}=40.9-65.4 \%)$ who had seen a mental health service provider in the past 6 months screening negative. Overall, 69.2\% $(n=101 / 146$; 95\% CI $=61.3-76.1 \%)$ who fell into one or more of these four groups were not considered to be at risk of depression according to the PHQ9, which accounts for $33.7 \%(n=101 / 300$; $95 \%$ CI $=28.6-$ $39.2 \%)$ of the total number of negative screens.

In terms of treatment, only $56.3 \%(n=67 / 119 ; 95 \%$ $\mathrm{CI}=47.3-64.9 \%)$ who self-reported depression and $78.6 \%$ $(n=33 / 42 ; 95 \% \mathrm{CI}=63.9-88.5 \%)$ who reported depression and screened positive used antidepressants and/or had seen a mental health service provider in the past 6 months.

3.4. Predictors of Depression Risk. Preliminary analyses of intercorrelation indicated a strong and significant association between self-reported depression and previous diagnosis of depression ( $r=0.935)$, resulting in these items being combined for further analyses. Along with the covariates, age, and gender, nine variables significantly associated with a positive screen were included in a backward stepwise logistic regression to identify the most parsimonious predictive model. These included self-reported depression and/or depression diagnosis; anxiety diagnosis; other mood disorder diagnoses; other mental illness diagnoses; current use of antidepressants; discussion of mental health with local doctor; recent access of a mental health service provider; smoking; and family history of anxiety. Discharge diagnoses were excluded from the model due to sample size constraints, and self-reported liver disease was excluded due to being a likely artefact of sampling.

After eight steps, four variables were retained in the final model, meeting the rule of thumb for logistic regression of 10 cases per predictor variable. Of those retained, self-reported depression and/or depression diagnosis ( $\mathrm{OR}=8.345 ; 95 \%$ $\mathrm{CI}=3.524-19.762 ; P<0.001)$, discussing mental health with a local doctor $(\mathrm{OR}=2.369 ; 95 \% \mathrm{CI}=1.025-5.475 ; P=0.044)$, and seeing a mental health service provider in the past 6 months $(\mathrm{OR}=4.518 ; 95 \% \mathrm{CI}=2.107-9.690 ; P<0.001)$ were independent predictors of depression risk.

This model had a strong goodness of fit (Hosmer and Lemeshow: $\chi^{2}=4.978 ; P=0.547$ ) and correctly classified $89.4 \%$ of participants. The variance accounted for by the final model ranged between $24.2 \%$ (Cox and Snell's $R$ square) and $43.3 \%$ (Nagelkerke's $R$ square).

\section{Discussion}

Research and information on ED-based depression screening in Australia and internationally are scarce. Most research in this area focuses on associated risk factors. This is the first study to assess depression risk in an Australian ED setting, document a prevalence of depression risk, and implement the PHQ-9 as an ED screening tool.

The total prevalence of ED patients screening positive for depression in this study was $14.3 \%(n=50 / 350$; $95 \% \mathrm{CI}=$ 11.0-18.4\%), more than twice that of the Australian general population (6\%) [6]. Notably, the PHQ-9 assesses over a two-week period while the national surveys obtained data over one month and twelve months; our results therefore are likely to underestimate the true prevalence. The prevalence was, however, lower than depression risk documented for ED settings internationally (22-32\%) [8-10] and in an Australian study of patients who reported feelings of anxiety or depression (20\%) [14]. Differences in exclusion criteria may account for this; unlike other studies, we excluded known high-risk [1, 26] patients who were agitated, intoxicated, affected by drugs, or presented with self-harm. The true prevalence of depression risk in the ED setting is therefore likely to be higher than that measured here. Additionally, the exclusion of a large proportion of those unable to communicate in English $(n=$ $271 / 831 ; 32.6 \%)$ may have affected our prevalence estimates. While rating scales are available in other main languages, their use alone without interpreters and a system of care and referral would be problematic and thus was not explored in this initial study. 
TABLE 3: Demographic and clinical characteristics for sample population (data are presented as number and percentage unless otherwise specified).

\begin{tabular}{|c|c|}
\hline & Total \\
\hline \multicolumn{2}{|l|}{ Demographic } \\
\hline Gender (male) & $184(52.6 \%)$ \\
\hline Age, median (IQR) & $49.0(31.5-66.5)$ \\
\hline \multicolumn{2}{|l|}{ Country of birth } \\
\hline Australia & $248(70.9 \%)$ \\
\hline Europe & $60(17.1 \%)$ \\
\hline Asia & $22(6.3 \%)$ \\
\hline Middle East and North Africa & $8(2.3 \%)$ \\
\hline Oceania & $7(2.0 \%)$ \\
\hline North America & $2(0.6 \%)$ \\
\hline Sub-Saharan Africa & $2(0.6 \%)$ \\
\hline Unknown & $1(0.3 \%)$ \\
\hline \multicolumn{2}{|l|}{ Marital status } \\
\hline Married & $134(38.3 \%)$ \\
\hline Single/never married & $131(37.4 \%)$ \\
\hline Widowed & $33(9.4 \%)$ \\
\hline Separated/divorced & $32(9.1 \%)$ \\
\hline De facto & $17(4.9 \%)$ \\
\hline Unknown & $3(0.9 \%)$ \\
\hline $\begin{array}{l}\text { Socioeconomic status (concession card } \\
\text { holder) }\end{array}$ & $121(34.6 \%)$ \\
\hline Mode of arrival (ambulance) & $150(42.9 \%)$ \\
\hline \multicolumn{2}{|l|}{ Triage score } \\
\hline 1 & $10(2.9 \%)$ \\
\hline 2 & $44(12.6 \%)$ \\
\hline 3 & $139(39.7 \%)$ \\
\hline 4 & $138(39.4 \%)$ \\
\hline 5 & $19(5.4 \%)$ \\
\hline Modal presenting complaint & Abdominal pain \\
\hline Modal discharge diagnosis & $\begin{array}{l}\text { Musculoskeletal } \\
\text { fracture/injury }\end{array}$ \\
\hline ED length of stay, median (IQR) & $297(193-456)$ \\
\hline \multicolumn{2}{|l|}{ SCQ } \\
\hline Heart disease & $70(20.0 \%)$ \\
\hline Hypertension & $102(29.1 \%)$ \\
\hline Lung disease & $82(23.4 \%)$ \\
\hline Diabetes & $38(10.9 \%)$ \\
\hline Ulcer or stomach disease & $95(27.1 \%)$ \\
\hline Kidney disease & $44(12.6 \%)$ \\
\hline Liver disease & $20(5.7 \%)$ \\
\hline Anaemia or other blood diseases & $56(16.0 \%)$ \\
\hline Cancer & $49(14.0 \%)$ \\
\hline Depression & $119(34.0 \%)$ \\
\hline Osteoarthritis or degenerative arthritis & $110(31.4 \%)$ \\
\hline Back pain & $173(49.4 \%)$ \\
\hline Rheumatoid arthritis & $17(4.9 \%)$ \\
\hline Other medical problems & $210(60.0 \%)$ \\
\hline
\end{tabular}

TABLE 3: Continued.

\begin{tabular}{lc}
\hline & Total \\
\hline Personal and family history survey & \\
Depression diagnosis & $114(32.6 \%)$ \\
Anxiety diagnosis & $123(35.1 \%)$ \\
Other mood disorder diagnoses & $6(1.7 \%)$ \\
Other mental illness diagnoses & $7(2.0 \%)$ \\
Current use of antidepressants & $54(15.4 \%)$ \\
Discussion of mental health with local & $136(38.9 \%)$ \\
doctor & \\
Seen mental health service provider in & $60(17.1 \%)$ \\
past 6 months & \\
Smoking & \\
$\quad$ Current & $83(23.7 \%)$ \\
$\quad$ Former & $59(16.9 \%)$ \\
Alcohol & $212(60.6 \%)$ \\
Family history of depression & $121(34.6 \%)$ \\
Family history of anxiety & $83(23.7 \%)$ \\
Family history of other mood & $35(10.0 \%)$ \\
disorders & \\
Family history of other mental illnesses & $39(11.1 \%)$ \\
\hline
\end{tabular}

TABLE 4: Classification of participant PHQ-9 screen.

\begin{tabular}{lcc}
\hline Classification & $\%$ & $95 \%$ CI \\
\hline Negative (0-4) & 54.3 & $49.1-59.4$ \\
Mild depression (5-9) & 31.4 & $26.8-36.5$ \\
Moderate depression (10-14) & 6.6 & $4.4-9.7$ \\
Moderately severe depression & 3.4 & $1.9-6.0$ \\
(15-19) & 4.3 & $2.6-7.0$ \\
\hline
\end{tabular}

The fact that the tool did not identify many patients with a suspected risk of depression based on self-reported depression, previous diagnoses of depression, current antidepressant use, and recent access to mental health services suggests that we may have underestimated the true prevalence of depression in this population. More than two-thirds of these patients did not screen positive on the PHQ-9, accounting for over a third of total negative screens. Although these patients may not have been presently depressed, due to the effects of treatment or natural remission of depressive illness, depressive disorders (notably major depressive disorder) tend to be chronic relapsing/remitting illnesses, so that these patients remain at ongoing risk. If all these participants were considered to have a risk of depression, this could mean that at least $40 \%$ of the ED population have depression risk.

Asking a patient whether they are currently suffering from depression could identify a majority of patients who may need help. Of participants who screened positive, $84 \%$ self-reported depression and $82 \%$ had previously been diagnosed with depression, suggesting that those who screen positive on the PHQ-9 are likely to be depressed. However, only $56 \%$ who self-reported depression and $79 \%$ who reported depression and screened positive used antidepressants and/or had seen a mental health service provider in the past 6 
TABLE 5: Association of clinical and demographic factors to the outcome of the PHQ-9 (data are number and percentage unless otherwise specified).

\begin{tabular}{|c|c|c|c|}
\hline & Negative screen & Positive screen & $P$ value \\
\hline \multicolumn{4}{|l|}{ Variable } \\
\hline Age, median (IQR) & $51.0(33.0-69.0)$ & $42.0(30.5-60.0)$ & 0.066 \\
\hline Gender (male) & $158(52.7 \%)$ & $26(52.0 \%)$ & 1.000 \\
\hline Country of birth (Australia) & $208(69.3 \%)$ & $40(80.0 \%)$ & 0.552 \\
\hline \multicolumn{4}{|l|}{ Marital status } \\
\hline Married & $117(39.0 \%)$ & $17(34.0 \%)$ & \multirow{6}{*}{0.293} \\
\hline Single/never married & $107(35.7 \%)$ & $24(48.0 \%)$ & \\
\hline Widowed & $32(10.7 \%)$ & $1(2.0 \%)$ & \\
\hline Separated/divorced & $27(9.0 \%)$ & $5(10.0 \%)$ & \\
\hline De facto & $14(4.7 \%)$ & $3(6.0 \%)$ & \\
\hline Unknown & $3(1.0 \%)$ & $0(0.0 \%)$ & \\
\hline Concession card holder & $106(35.3 \%)$ & $15(30.0 \%)$ & 0.523 \\
\hline Arrival mode (ambulance) & $129(43.0 \%)$ & $21(42.0 \%)$ & 1.000 \\
\hline \multicolumn{4}{|l|}{ Triage category } \\
\hline 1 & $9(3.0 \%)$ & $1(2.0 \%)$ & \multirow{5}{*}{0.545} \\
\hline 2 & $36(12.0 \%)$ & $8(16.0 \%)$ & \\
\hline 3 & $122(40.7 \%)$ & $17(34.0 \%)$ & \\
\hline 4 & $115(38.3 \%)$ & $23(46.0 \%)$ & \\
\hline 5 & $18(6.0 \%)$ & $1(2.0 \%)$ & \\
\hline Presenting complaint (abdominal pain) & $43(14.3 \%)$ & $5(10.0 \%)$ & 0.410 \\
\hline Diagnosis (musculoskeletal) & $67(22.3 \%)$ & $9(18.0 \%)$ & $<0.001$ \\
\hline ED length of stay, median (IQR) & $296.0(189.3-450.8)$ & $301.5(211.0-487.5)$ & 0.440 \\
\hline Further admission & $222(74.0 \%)$ & $35(70.0 \%)$ & 0.604 \\
\hline \multicolumn{4}{|l|}{ SCQ } \\
\hline Heart disease & $61(20.3 \%)$ & $9(18.0 \%)$ & 0.849 \\
\hline High blood pressure & $89(29.7 \%)$ & $13(26.0 \%)$ & 0.737 \\
\hline Lung disease & $68(22.7 \%)$ & $14(28.0 \%)$ & 0.470 \\
\hline Diabetes & $33(11.0 \%)$ & $5(10.0 \%)$ & 1.000 \\
\hline Ulcer or stomach disease & $79(26.3 \%)$ & $16(32.0 \%)$ & 0.396 \\
\hline Kidney disease & $37(12.3 \%)$ & $7(14.0 \%)$ & 0.817 \\
\hline Liver disease & $10(3.3 \%)$ & $10(20.0 \%)$ & $<0.001$ \\
\hline Anaemia or other blood conditions & $44(14.7 \%)$ & $12(24.0 \%)$ & 0.099 \\
\hline Cancer & $44(14.7 \%)$ & $5(10.0 \%)$ & 0.510 \\
\hline Depression & $77(25.7 \%)$ & $42(84.0 \%)$ & $<0.001$ \\
\hline Osteo/degenerative arthritis & $96(32.0 \%)$ & $14(28.0 \%)$ & 0.625 \\
\hline Back pain & $143(47.7 \%)$ & $30(60.0 \%)$ & 0.127 \\
\hline Rheumatoid arthritis & $15(5.0 \%)$ & $2(4.0 \%)$ & 1.000 \\
\hline Other medical problems & $178(59.3 \%)$ & $32(64.0 \%)$ & 0.640 \\
\hline \multicolumn{4}{|l|}{ Personal and family history survey } \\
\hline Depression diagnosis & $73(24.3 \%)$ & $41(82.0 \%)$ & $<0.001$ \\
\hline Other mood disorder diagnoses & $3(1.0 \%)$ & $3(6.0 \%)$ & 0.040 \\
\hline Anxiety diagnosis & $88(29.3 \%)$ & $35(70.0 \%)$ & $<0.001$ \\
\hline Other mental illness diagnoses & $3(1.0 \%)$ & $4(8.0 \%)$ & 0.009 \\
\hline Use of antidepressant & $33(11.0 \%)$ & $21(42.0 \%)$ & $<0.001$ \\
\hline Discuss mental health with local doctor & $97(32.3 \%)$ & $39(78.0 \%)$ & $<0.001$ \\
\hline Seen mental health service provider in past 6 months & $32(10.7 \%)$ & $28(56.0 \%)$ & $<\mathbf{0 . 0 0 1}$ \\
\hline \multicolumn{4}{|l|}{ Smoking } \\
\hline Current & $61(20.3 \%)$ & $22(44.0 \%)$ & \multirow{2}{*}{0.001} \\
\hline Former & $56(18.7 \%)$ & $3(6.0 \%)$ & \\
\hline Alcohol use & $182(60.7 \%)$ & $30(60.0 \%)$ & 1.000 \\
\hline Family history of depression & $98(32.7 \%)$ & $23(46.0 \%)$ & 0.091 \\
\hline Family history of anxiety & $63(21.0 \%)$ & $20(40.0 \%)$ & $<0.001$ \\
\hline Family history of other mood disorders & $26(8.7 \%)$ & $9(18.0 \%)$ & 0.056 \\
\hline Family history of other mental illnesses & $31(10.3 \%)$ & $8(16.0 \%)$ & 0.164 \\
\hline
\end{tabular}


months. This highlights that treatment among high-risk ED patients may be improved through screening and linkage to appropriate services. In the busy emergency department, clinicians may be reluctant to undertake universal screening for conditions unrelated to the presenting complaint. Further study into methods by which to facilitate screening may be warranted.

Our study did not replicate previous findings of an association of depression risk with increasing age [9] or heart disease [5]. The reasons for this are unclear. Family history of psychiatric disturbance, including depression, was also not shown to be significant. As it was difficult to obtain medical diagnoses of family members, we relied heavily on the participant's report. This may not have been accurate, as some participants were disconnected from their families and were unaware of any family history of mental health disorders, while others suspected a history but were not aware of any formal diagnosis.

4.1. Limitations. We did not use a gold standard (such as structured psychiatric interview) to verify diagnoses of depression and therefore cannot claim validity of the PHQ9 in an ED setting.

Due to the limited time availability of the sole recruiting researcher, a consecutive sample was not obtained. The ED operates for 24 hours per day, and thus the sample is likely not to adequately represent patients attending outside sampling hours. The exclusion of those who were cognitively impaired, drug or alcohol affected, or were presented with deliberate self-harm resulted in a large number of patients being excluded from the study, affecting the representativeness of the sample and most likely resulting in an underestimate of depression risk.

4.2. Future Research and Directions. ED-based depression screening could yield an overall benefit to the general population and the healthcare system. However ideally there should be a tool validated specifically for the ED. This study was powered to estimate prevalence to within a 5\% level of precision in the overall sample. In order to more reliably determine the prevalence of depression within subgroups, future studies should employ a larger sample enabling stratification for age and other key clinical and demographic factors, whilst retaining precision within each group. Depression screening in non-English speakers through the use of translatable tools and interpreters also deserves further exploration, as they form a substantial proportion of the ED population. In addition, a cost-benefit analysis should be performed to assess the acceptance of depression screening among patients and staff and determine how well ED doctors refer those who screen positive. This will allow us to predict the feasibility and practicality of routine depression screening in an ED setting. Most EDs operate 24 hours per day, so future studies need to be adequately resourced to sample subjects overnight.

\section{Conclusion}

We have shown that ED patients are at considerably higher risk of having depression than the general population, even allowing for high-risk groups excluded from this study. From a clinical perspective, although most cases of depression can be ascertained by simple clinical interview, using questions about current mood and past psychiatric history, an important and substantial subset failed to be detected by routine ED assessment. Thus depression screening needs to be developed and evaluated as a potentially valuable complement to ordinary clinical assessment. The ED is a health system setting which can make a very useful contribution to reducing the population burden of depression.

\section{Conflict of Interests}

The authors declare that they have no conflict of interests.

\section{Authors' Contribution}

Tracey J. Weiland and George A. Jelinek conceived the study. Nancy Khav, Tracey J. Weiland, George A. Jelinek, and Michael Salzberg designed the methodology. Nancy Khav and Tracey J. Weiland obtained research funding and ethics approval. Tracey J. Weiland, George A. Jelinek, and Jonathan C. Knott supervised the conduct of the study and data collection. Nancy Khav undertook recruitment of participants and managed the data. Tracey J. Weiland, George A. Jelinek, and Jonathan C. Knott provided statistical advice on the study design. Nancy Khav and Tracey J. Weiland analysed the data. Nancy Khav drafted the paper, and all the authors contributed substantially to its revision. Nancy Khav takes responsibility for the paper as a whole.

\section{Acknowledgment}

This study was financially supported by the University of Melbourne, VIC, Australia.

\section{References}

[1] A. Mackinnon, A. F. Jorm, and I. B. Hickie, "A National Depression Index for Australia," Medical Journal of Australia, vol. 181, no. 7, supplement, pp. S52-S56, 2004.

[2] R. C. Kessler, P. Berglund, O. Demler et al., "The epidemiology of major depressive disorder. Results from the National Comorbidity Survey Replication (NCS-R)," Journal of the American Medical Association, vol. 289, no. 23, pp. 3095-3105, 2003.

[3] C. J. L. Murray and A. D. Lopez, "Alternative projections of mortality and disability by cause 1990-2020. Global Burden of Disease Study," Lancet, vol. 349, no. 9064, pp. 1498-1504, 1997.

[4] M. R. DiMatteo, H. S. Lepper, and T. W. Croghan, "Depression is a risk factor for noncompliance with medical treatment metaanalysis of the effects of anxiety and depression on patient adherence," Archives of Internal Medicine, vol. 160, no. 14, pp. 2101-2107, 2000.

[5] J. H. Lichtman, J. T. Bigger Jr., J. A. Blumenthal et al., "Depression and coronary heart disease: recommendations for screening, referral, and treatment-a science advisory from the American Heart Association Prevention Committee of the Council on Cardiovascular Nursing, Council on Clinical Cardiology, Council on Epidemiology and Prevention, and Interdisciplinary Council on Quality of Care and Outcomes Research," Circulation, vol. 118, no. 17, pp. 1768-1775, 2008. 
[6] I. B. Hickie, T. A. Davenport, E. M. Scott, D. Hadzi-Pavlovic, S. L. Naismith, and A. Koschera, "Unmet need for recognition of common mental disorders in Australian general practice," Medical Journal of Australia, vol. 175, supplement 2, pp. S18-S24, 2001.

[7] “Beyond Blue. Depression. Beyond Blue, 2007,” 2011, http:// www.beyondblue.org.au/index.aspx?link_id=89.578.

[8] E. D. Boudreaux, S. Clark, and C. A. Camargo Jr., "Mood disorder screening among adult emergency department patients: a multicenter study of prevalence, associations and interest in treatment," General Hospital Psychiatry, vol. 30, no. 1, pp. 4-13, 2008.

[9] E. D. Boudreaux, C. Cagande, H. Kilgannon, A. Kumar, and C. A. Camargo Jr., "A prospective study of depression among adult patients in an urban emergency department," Primary Care Companion to the Journal of Clinical Psychiatry, vol. 8, no. 2, pp. 66-70, 2006.

[10] D. Hoyer and E. David, "Screening for depression in emergency department patients," Journal of Emergency Medicine, vol. 43, no. 5, pp. 786-789, 2012.

[11] A. Kumar, S. Clark, E. D. Boudreaux, and C. A. Camargo Jr., "A multicenter study of depression among emergency department patients," Academic Emergency Medicine, vol. 11, no. 12, pp. 1284-1289, 2004.

[12] F. Perruche, C. Elie, M. D’Ussel et al., "Anxiety and depression are unrecognised in emergency patients admitted to the observation care unit," Emergency Medicine Journal, vol. 28, no. 8, pp. 662-665, 2011.

[13] D. L. Schriger, P. S. Gibbons, C. A. Langone, S. Lee, and L. L. Altshuler, "Enabling the diagnosis of occult psychiatric illness in the emergency department: a randomized, controlled trial of the computerized, self-administered PRIME-MD diagnostic system," Annals of Emergency Medicine, vol. 37, no. 2, pp. 132140, 2001.

[14] S. A. Kinner, R. Alati, K. Watt, J. M. Najman, G. Fowler, and D. Green, "Substance misuse, anxiety and depression and urgency of presentation to a public emergency department in Australia," Emergency Medicine Australasia, vol. 17, no. 4, pp. 363-370, 2005.

[15] M. Fry and S. Brunero, "The characteristics and outcomes of mental health patients presenting to an emergency department over a 12 month period," Australian Emergency Nursing Journal, vol. 7, pp. 21-25, 2004.

[16] J. C. Knott, A. Pleban, D. Taylor, and D. Castle, "Management of mental health patients attending Victorian emergency departments," Australian and New Zealand Journal of Psychiatry, vol. 41, no. 9, pp. 759-767, 2007.

[17] K. V. Rhodes, "Mood disorders in the emergency department: the challenge of linking patients to appropriate services," General Hospital Psychiatry, vol. 30, no. 1, pp. 1-3, 2008.

[18] C. L. Miller and B. Druss, "Datapoints: suicide and access to care," Psychiatric Services, vol. 52, no. 12, article 1566, 2001.

[19] L. Heslop, S. Elsom, and N. Parker, "Improving continuity of care across psychiatric and emergency services: combining patient data within a participatory action research framework," Journal of Advanced Nursing, vol. 31, no. 1, pp. 135-143, 2000.

[20] K. Kroenke and R. L. Spitzer, "The PHQ-9: a new depression diagnostic and severity measure," Psychiatric Annals, vol. 32, no. 9, pp. 509-515, 2002.

[21] K. Kroenke, R. L. Spitzer, and J. B. W. Williams, “The PHQ9: validity of a brief depression severity measure," Journal of General Internal Medicine, vol. 16, no. 9, pp. 606-613, 2001.
[22] B. Löwe, K. Kroenke, and K. Gräfe, "Detecting and monitoring depression with a two-item questionnaire (PHQ-2)," Journal of Psychosomatic Research, vol. 58, pp. 163-171, 2005.

[23] O. Sangha, G. Stucki, M. H. Liang, A. H. Fossel, and J. N. Katz, "The Self-Administered Comorbidity Questionnaire: a new method to assess comorbidity for clinical and health services research," Arthritis Care and Research, vol. 49, no. 2, pp. 156163, 2003.

[24] L. Naing, T. Winn, and B. N. Rusli, "Practical issues in calculating the sample size for prevalence studies," Archives of Orofacial Sciences, vol. 1, pp. 9-14, 2006.

[25] T. Slade, A. Johnston, M. A. Oakley Browne, G. Andrews, and H. Whiteford, "2007 National Survey of Mental Health and Wellbeing: methods and key findings," Australian and New Zealand Journal of Psychiatry, vol. 43, no. 7, pp. 594-605, 2009.

[26] I. B. Hickie, A. Koschera, T. A. Davenport, S. L. Naismith, and E. M. Scott, "Comorbidity of common mental disorders and alcohol or other substance misuse in Australian general practice," Medical Journal of Australia, vol. 175, supplement 2, pp. S31-S36, 2001. 


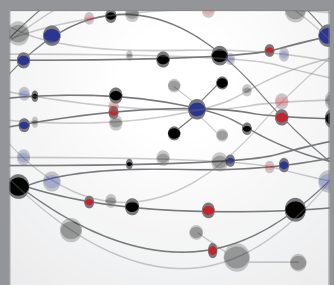

The Scientific World Journal
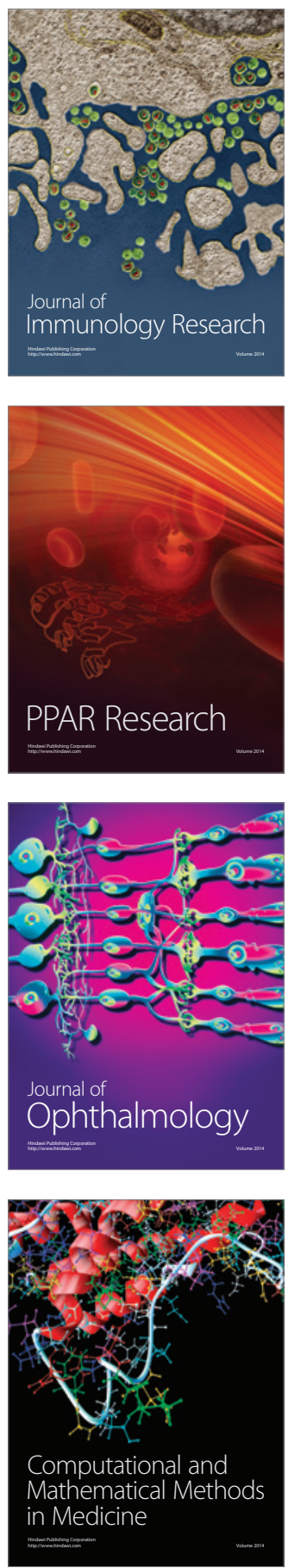

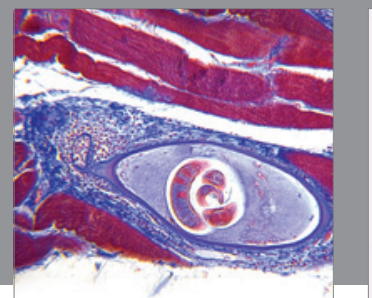

Gastroenterology

Research and Practice
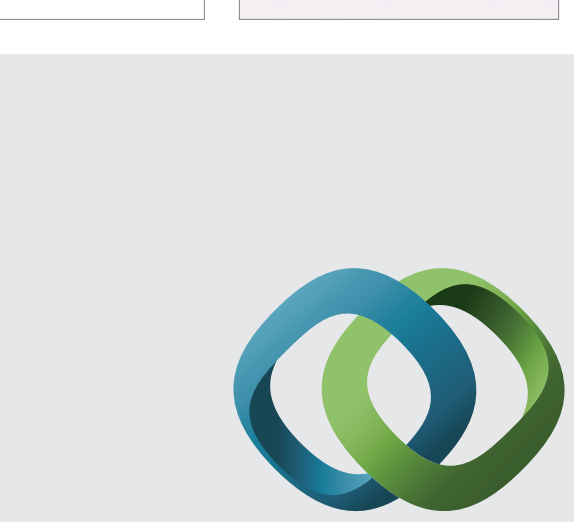

\section{Hindawi}

Submit your manuscripts at

http://www.hindawi.com
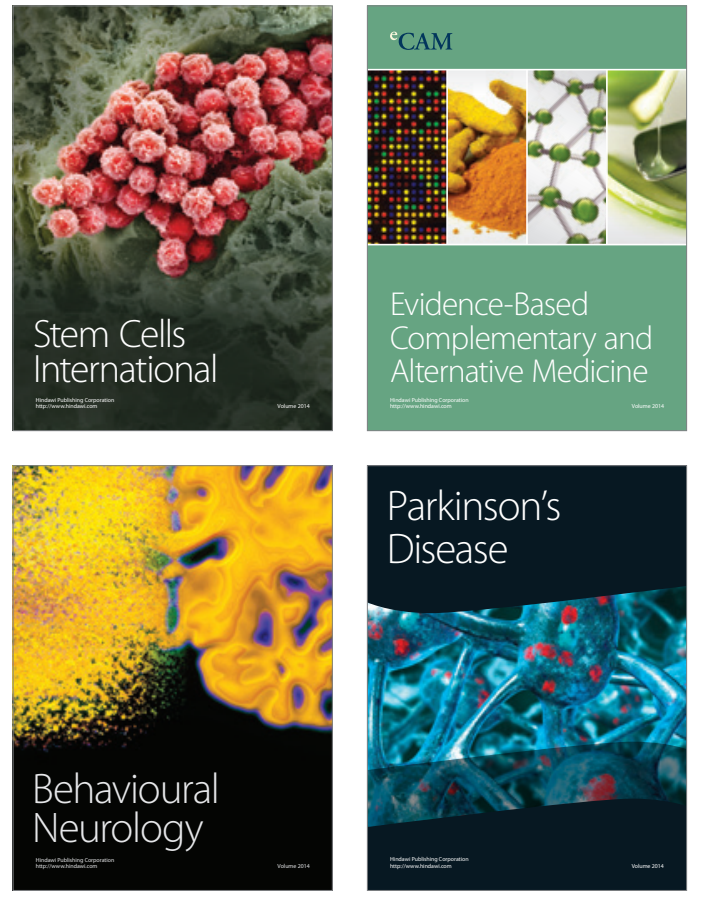
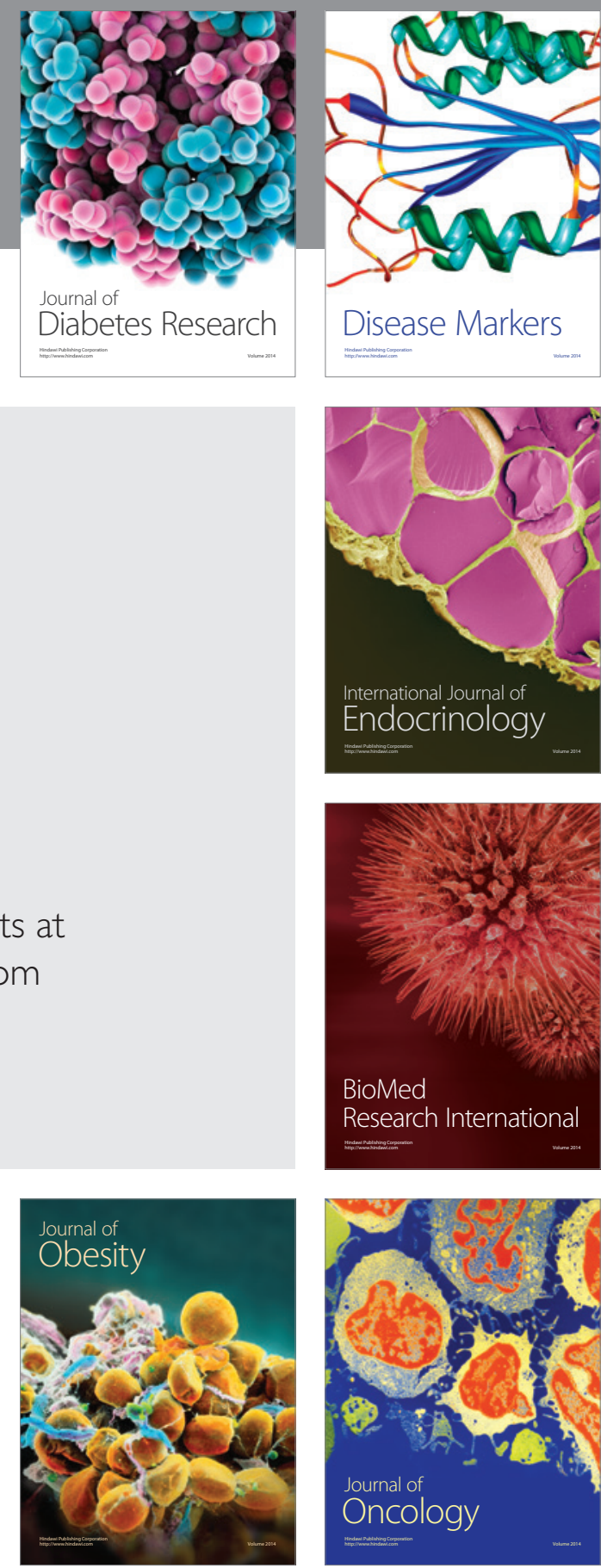

Disease Markers
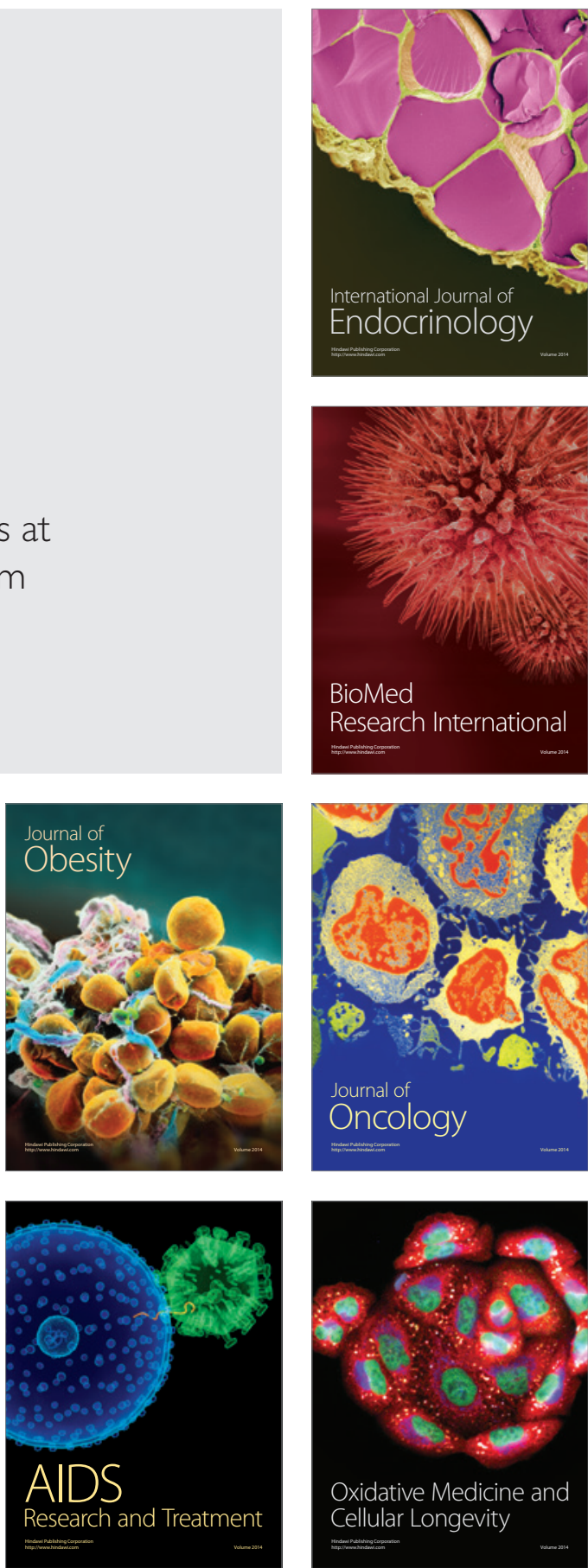